\title{
The VAWT in Skew: Stereo-PIV and Vortex Modeling
}

\author{
Carlos Ferreira ${ }^{*}$ Kristian Dixon † Claudia Hofemann \\ Gijs van Kuik § Gerard van Bussel ๆ \\ Delft University of Technology, Delft, 2629 HS, The Netherlands
}

\begin{abstract}
One of the results of the development of wind energy conversion solutions for the built environment is the reappearance of Vertical Axis Wind Turbines (VAWTs).

The application of wind turbines in urban environments presents design challenges driven by the complex wind fields experienced in the urban boundary layer. Urban Wind Turbines operate near, on and in the wake of bluff bodies larger than the rotor scale. These flow conditions might result in skewed flow operation.

The objective of the current paper is to bring insight into the development of the near wake of a H-VAWT in skewed flow, namely understanding:

- Blade loading asymmetry in spanwise direction.

- Trajectory of the tip vortices, including inboard movement and radial expansion of the shed and trailing vorticity.

- Asymmetry of the wake in spanwise direction.

- Blade vortex interaction of upwind tip vortex with downwind blade passage.

- Load distribution in downwind blade passage.

- Effect of skew in the expansion of the mid-wake.

The investigation is composed of experimental wind tunnel research of a two bladed H-Darrieus VAWT model with Particle Image Velocimetry, and modeling of the rotor and wake with a $3 D$ unsteady panel method.

Simulations of the rotor in skewed flow are validated with the experimental PIV data and with the torque measurements of ${ }^{1}$ and ${ }^{2}$.

The results of the panel model, validated by experiments, show the impact of skew angle on the near wake's development, both for the upwind blade passage as well as the downwind blade passage.
\end{abstract}

\section{Nomenclature}

$c \quad$ airfoil/blade chord, $m$

$R \quad$ rotor radius, $m$

$H \quad$ rotor height, $m$

$F_{N} \quad$ Normal force, $N$

$F \quad$ Force, $N$

$U_{\infty} \quad$ Unperturbed velocity $\mathrm{m} / \mathrm{s}$

$U_{x} \quad$ Local velocity in $x$-direction $\mathrm{m} / \mathrm{s}$

$U_{\text {ind }(x)} \quad$ Induced velocity in $x$-direction $\mathrm{m} / \mathrm{s}$

$U_{\text {ind }(z)} \quad$ Induced velocity in $z$-direction $\mathrm{m} / \mathrm{s}$

$x, y, z \quad$ coordinates $\mathrm{m} / \mathrm{s}$

$\beta \quad$ skew angle

${ }^{*}$ DUWIND, TUDelft, The Netherlands.

${ }^{\dagger}$ DUWIND, TUDelft, The Netherlands

¥DUWIND, TUDelft, The Netherlands.

$\S$ Professor, DUWIND, TUDelft, The Netherlands.

IProfessor, DUWIND, TUDelft, Kluyverweg 1, 2629 HS, Delft, The Netherlands 


\section{Introduction}

The application of wind turbines in urban environments presents design challenges driven by the complex wind fields experienced in the urban boundary layer. Urban Wind Turbines operate near, on and in the wake of bluff bodies larger than the rotor scale. These flow conditions might result in skewed flow operation. Skewed flow is, similarly to yawed flow, a misalignment between wind direction and the normal to the actuator disk representing the rotor. While yaw consists of a misalignment in the plane parallel to the ground, skewed flow relates to the misalignment component in the plane perpendicular to the ground. Skewed flow is thus not likely to occur in large scale Horizontal Axis Wind Turbines in open fields; yet, in the roof of buildings and in the urban boundary layer, it affects significantly the performance of the turbine $\left({ }^{2}\right)$.

One of the results of the development of solutions for the built environment is the reappearance of Vertical Axis Wind Turbines (VAWTs). In the built environment, the VAWT presents several advantages over the more common Horizontal Axis Wind Turbines (HAWTs), namely: its low sound emission (consequence of its operation at lower tip speed ratios), better esthetics due to its three-dimensionality, its insensitivity to yaw wind direction and its increased power output in skewed flow $\left(\mathrm{see}^{2}\right.$ and $\left.{ }^{3}\right)$.

The physics behind this increased performance of the VAWT in skewed flow are the driver of the current research. In non-skewed flow, the downwind half of the rotation of the VAWT blade operates in the wake of the upwind blade passage, thus in flow with a lower energy content. In skewed flow, the downwind passage of the wake is only partially in the wake of the upwind passage. Skewed flow, in a simplified approach, results in an increase of effective surface area of the rotor, although at a skewed direction, which implies a loss of power similar to the effect of yaw.

In this work, we will evaluate the effect of skewed flow on a H-Darrieus, observing:

- The impact of skew as a source of asymmetry on the strength and location of the near wake.

- The variation of torque with skewed angle, considering the contributions of upwind and downwind halves of the rotation.

To analyze the impact of skewed flow, we use two tools:

- Particle Image Velocimetry (PIV) of the tip vortex of the VAWT in skewed flow

- 3D unsteady free wake panel method simulations of the VAWT in skewed flow

The PIV data of the tip vortices, which validates the results of the $3 D$ unsteady panel code, allows the evaluation of the effect of skew angle on the strength and convection of the tip vortex. The experimental and numerical results show a very good agreement with the PIV results. A description of the experimental setup and procedure is found in ${ }^{4}$ and. ${ }^{5}$

The $3 D$, unsteady, multi-body, free-wake panel method is intended as a design and research tool to capture the $3 D$ nature of a VAWT and its wake. The model contains methods to realistically treat blade-wake interactions, vortex stretching/contraction and viscous diffusion. Validation of the model is demonstrated in ${ }^{6}$ and ${ }^{7}$ by comparison with PIV and smoke trail studies for straight-bladed VAWTs.

The $3 D$ unsteady free wake panel method simulations permit the analysis of the effect of skew in terms of bound circulation, shed and trailing vorticity strength, torque, wake and flow asymmetry in $z$ direction.

The panel model is also used to simulate the experimental work of ${ }^{2}$ and ${ }^{1}$ in relation to the effect of skewed flow on torque/power.

\section{Experimental Investigation with PIV}

The wind tunnel experimental work was conducted in the TUDelft's Low-Speed Low-Turbulence Wind Tunnel; the experimental setup and the equipment used is described $i^{4}{ }^{4}$ and. ${ }^{5}$ 
The experimental model is a two bladed H-VAWT. The two blades are $0.7 \mathrm{~m}$ span and $0.06 \mathrm{~m}$ chord, one with a NACA 0018 profile and the other a NACA 0015. The two blades have four different tip shapes to evaluate the effect of the tip shape on the tip vortex strength and location. The experimental measurements where conducted at a tip speed ratio $\lambda=4$ and Reynolds number approximately $8 * 10^{4}$. The results presented in this paper regard the tip vortex released from the blade tip with an elliptic chord distribution and a straight trailing edge $\left(\right.$ see $\left.^{4}\right)$. Figure 1 shows a schematic of the VAWT model in the wind tunnel, including laser sheet and acquisition cameras.

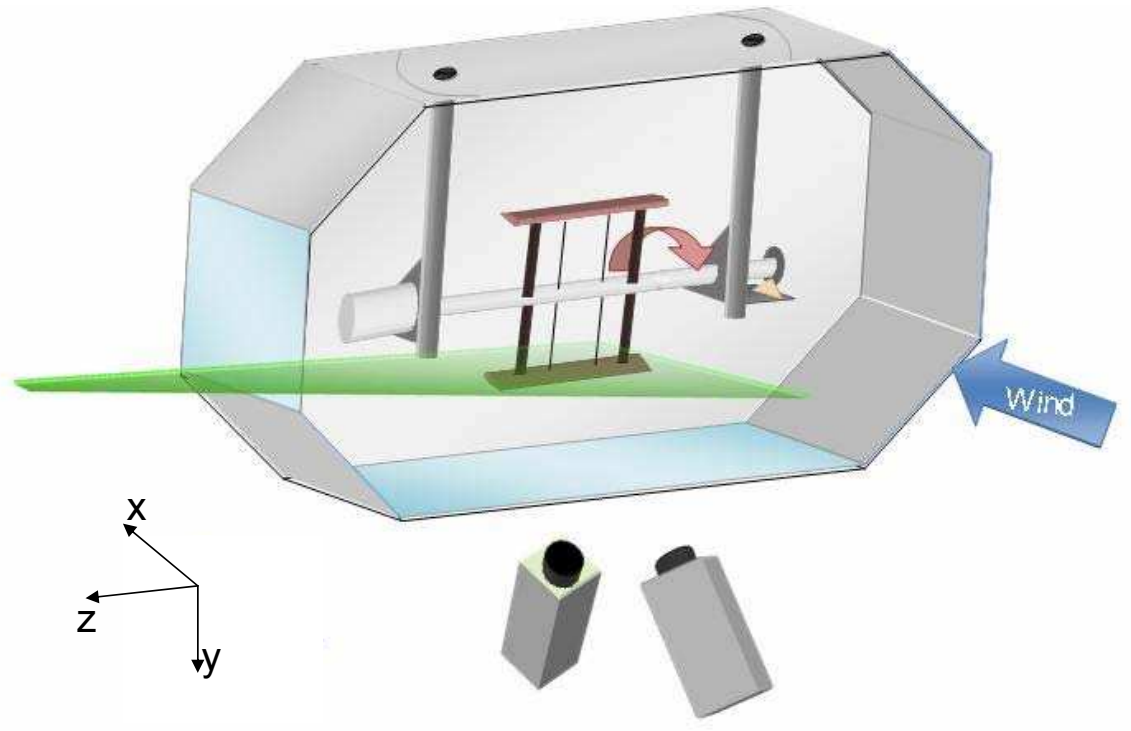

Figure 1: Schematic of the experimental setup, including rotor model, wind tunnel, laser sheet and acquisition cameras.

The experimental results presented refer to the tip vortex region. Figure 2 shows the location of the three fields of view of the PIV measurements, at three planes normal to $y$-direction: $y / R=-.42, y / R=0$ and $y / R=-.42$. The experimental setup and available wind tunnel time limited the available data in terms of downwind area. The data is then confined to the first third of the radius after the release point.

Figure 3a shows the location of the tip vortex core for three different skew angles $\beta=20^{\circ}, 0^{\circ}$ and $-20^{\circ}$ (around $y$ axis), on three planes normal to $y$-direction: $y / R=-.42, y / R=0$ and $y / R=-.42$. The trajectory lines do not start at the location of the tip vortex, since, due to the dynamics of the tip vortex generation, it is difficult to define the exact moment of release of the tip vortex; the authors have decided to plot only data referring to locations when the tip vortex is clearly detached from the blade, and the blade has passed the plane of the laser sheet.

The results of Figure 3a show that the convection of the tip vortex is dominated by the component of $U_{\infty}$ in $z$-direction. However, the subplot in Figure 3 a for $\beta=0^{\circ}$ shows clearly the effect of the rolling up process of the tip vortex and the induction of the remaining wake; the tip vortex does not displace with $U_{\infty}$, but move inboard $\left(\frac{2 z}{H}<1\right)$.

However, skewed flow will have an effect on the motion of the tip vortex in relation to $U_{\infty}$. Figure $3 \mathrm{~b}$ shows the trajectory of the tip vortices, in relation to $U_{\infty}$ (the trajectory is rotated by the skew angle, in relation to the point of intersection of the measurement plane with the blade tip).

The results in Figure 3b clearly show a difference in the relative motion of the vortices for the three skew angles. The relative trajectories for the cases $\beta=0^{\circ}$ and $\beta=-20^{\circ}$ are similar, both showing the relative inboard motion; however the trajectory for $\beta=20^{\circ}$ shows the opposite trend, having a more outboard motion.

There are two sources for the difference of relative trajectories of the tip vortices as a function of skew:

- The impact of skew in the shape, location and strength of the wake, and thus in the induction/local velocity experienced by the tip vortex. 


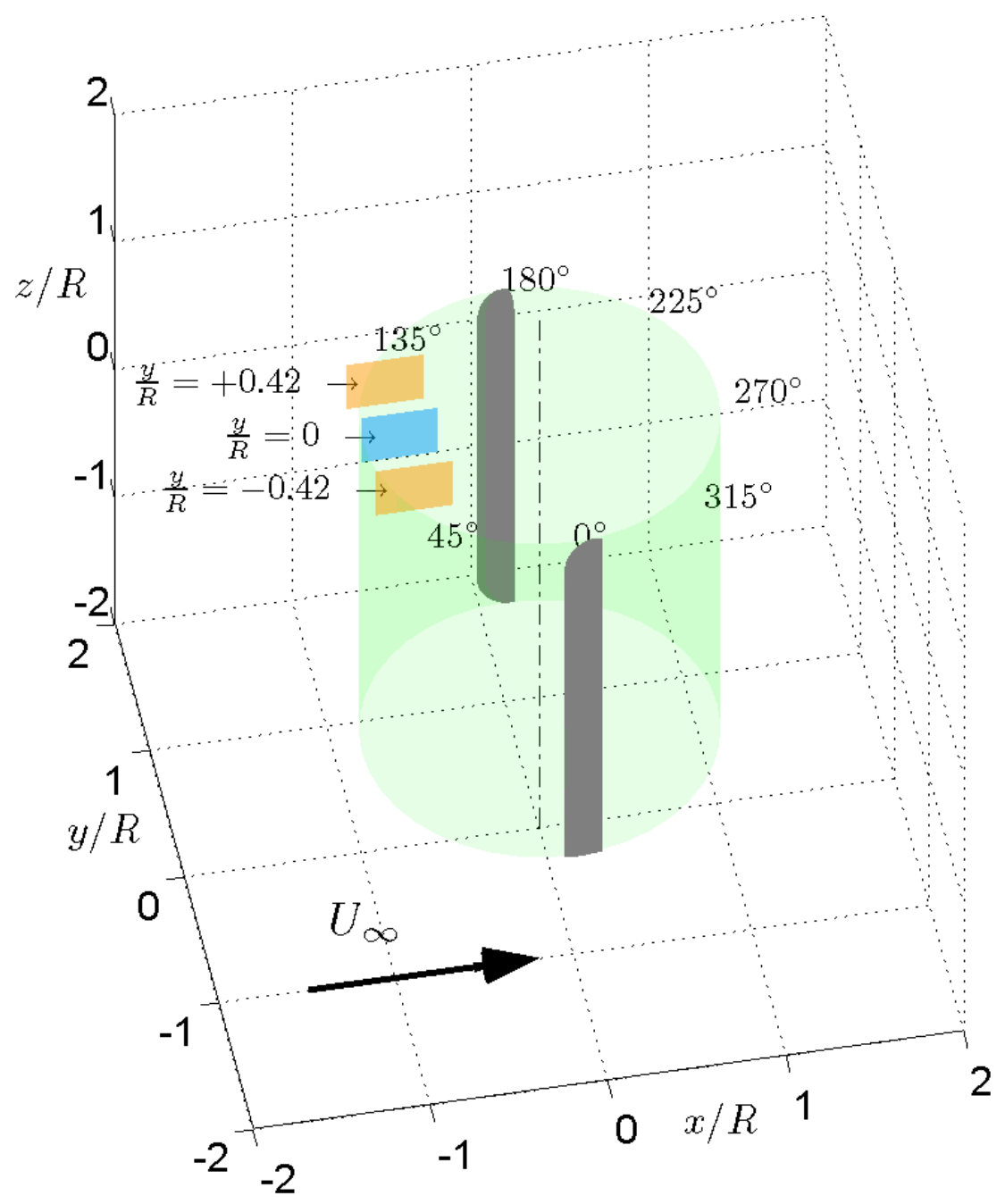

Figure 2: Schematic of the rotor, including fields of view at planes $\frac{y}{R}=-0.42,0$ and 0.42 . 


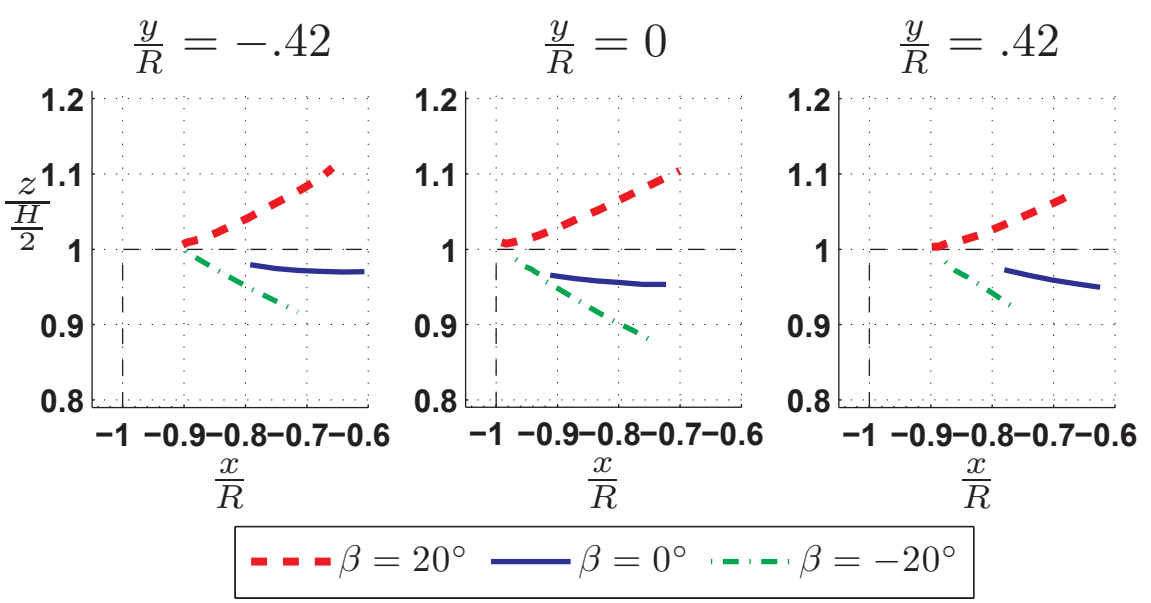

(a) Tip vortex locus (absolute).

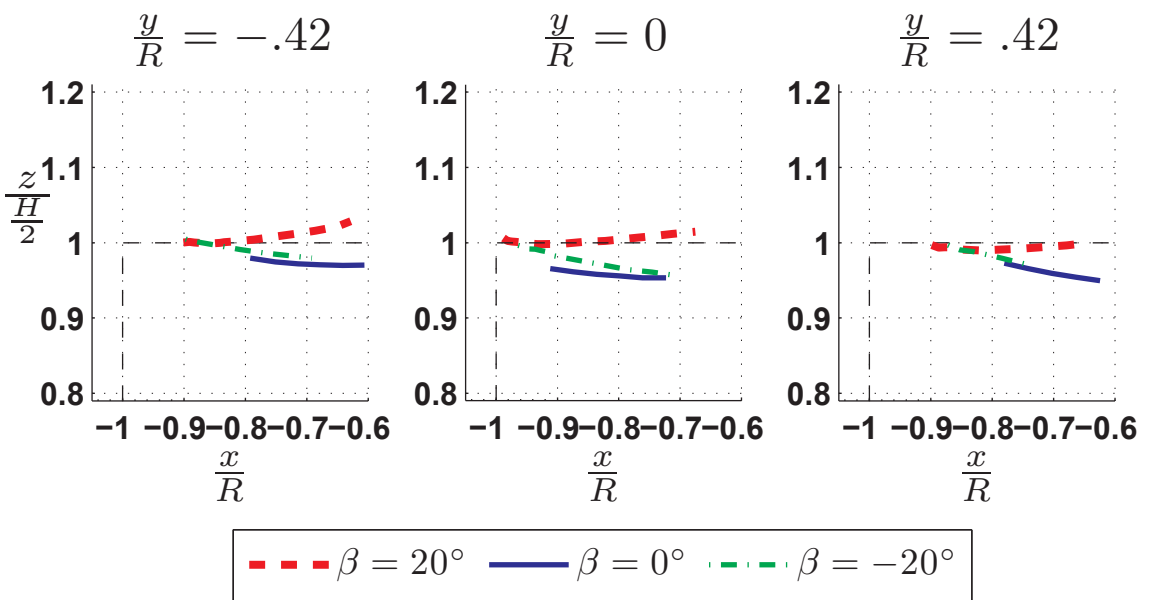

(b) Tip vortex locus relative to $U_{\infty}$ direction.

Figure 3: Tip vortex locus (experimental data) for $\beta=+20^{\circ}, 0^{\circ}$ and $-20^{\circ}$, at the planes $\frac{y}{R}=-.42,0$ and .42 . 
- the impact of skew in the strength of the tip vortex and nearby vorticity, which affects its roll up process; this is a result of the effect of skew on the induction experienced by the blade.

In the following sections, by using the experimental results and the numerical simulations, we see that both sources are at play and are aligned. For $\beta=0^{\circ}$ and $\beta=-20^{\circ}$, the strength of the tip vortex is similar (following sections of the paper), and thus, the roll-up process is also similar. The induction of the wake, although slightly different, is, for both angles, dominated by the near wake. We also see that, for $\beta=20^{\circ}$, due to the geometry of the wake, the induction in $x$-direction is larger, and thus, not only is the strength of the tip vortex smaller, also the tip vortex experiences an effectively larger skew angle on its trajectory.

\section{Position of the wake}

In the previous section, we discussed the effect of skew on the motion of the tip vortex. We will now use numerical simulations with a $3 D$ free wake panel code (see ${ }^{6}$ and ${ }^{7}$ ) to understand the effect of skew on the induction, convection, circulation and loads of the VAWT.

Figure 4 shows two views of the location of the wake (when $\theta=90^{\circ}$ ) for three skew angles $\beta=+20^{\circ}$, $0^{\circ}$ and $-20^{\circ}$ (numerical simulation). The two views show two clear effects of skew in the convection of the wake:

- The convection in $z$ direction of the wake.

- The asymmetry in $z$ direction of the expansion of the wake in $y$ direction.

The convection of the wake is mainly driven by the magnitude and direction of the unperturbed flow field $U_{\infty}$. The convection in $z$ direction of the wake implies an asymmetry in $z$ direction of the induction of the wake on the blade; we will discuss the impact of this asymmetry in Section V.

\section{Asymmetry of the induction in $z$}

The definition of skewed flow for a VAWT is the flow resulting from the operation of a VAWT in conditions that can be characterized by $U_{\infty}$ non-normal to the axis of rotation. One of the main effects of skewed flow, is that the main direction of convection of the wake is also not normal to the axis of rotation; this results in an asymmetry of the location of the wake in $z$ direction.

The asymmetry in $z$ of location of the wake leads to an asymmetry in $z$ direction of the velocity induced by the wake, both the induction on the blades as the self induction of the wake.

In this section we will discuss the effect of this asymmetry on the induction during the upwind passage of the blade. Figures 5 and 6 show respectively the $x$ and $z$ direction components of induction at the blade tip region, when the blade is at the most upwind position $\left(\frac{2 z}{H}=1, \frac{x}{R}=-1, \frac{y}{R}=0\right.$ and $\left.\theta=90^{\circ}\right)$, marked with a green dot. The color over the mesh of the wake indicates the velocity induced per unit length of vortex line.

Figures 5 and 6 present each six sub-plots, organized in two columns, where the left column shows the induction due to the trailing vorticity, and the right column shows the induction due to the shed vorticity. The three lines organize the results according to skew angle $\beta=+20^{\circ}, 0^{\circ}$ and $-20^{\circ}$.

\section{A. Induction due to trailing vorticity}

The skewed flow causes the wake to travel in $z$ direction, either upward $\left(\beta=20^{\circ}\right)$ or downward $\left(\beta=-20^{\circ}\right)$. The left column of Figure 5 shows the induction due to trailing vorticity.

A large contribution of induction comes from the trailing vorticity of the wake generated during the second blade's latest upwind passage. For this patch of wake, the sign of the induction (positive or negative in $x$ direction) of this trailing vorticity is a function of the location in $z$ of the trailing vortex filament, has the trailing vorticity has all the same sign close to the blade tip, for all three skew angles; the induction at $\frac{2 z}{H}=1, \frac{x}{R}=-1, \frac{y}{R}=0$ by a vortex filament is positive if the filament is located at $\frac{2 z}{H}<1$ and negative for $\frac{2 z}{H}>1$. In the non-skewed flow case, the induction is positive since the roll-up behavior of the tip vortex convects it to $\frac{2 z}{H}<1$.

This asymmetry with skewed angle of the velocity induced in $x$ direction explains also the difference in strength of the tip vortex. In the next sections, we will discuss further the impact in the strength of the tip vortex. 

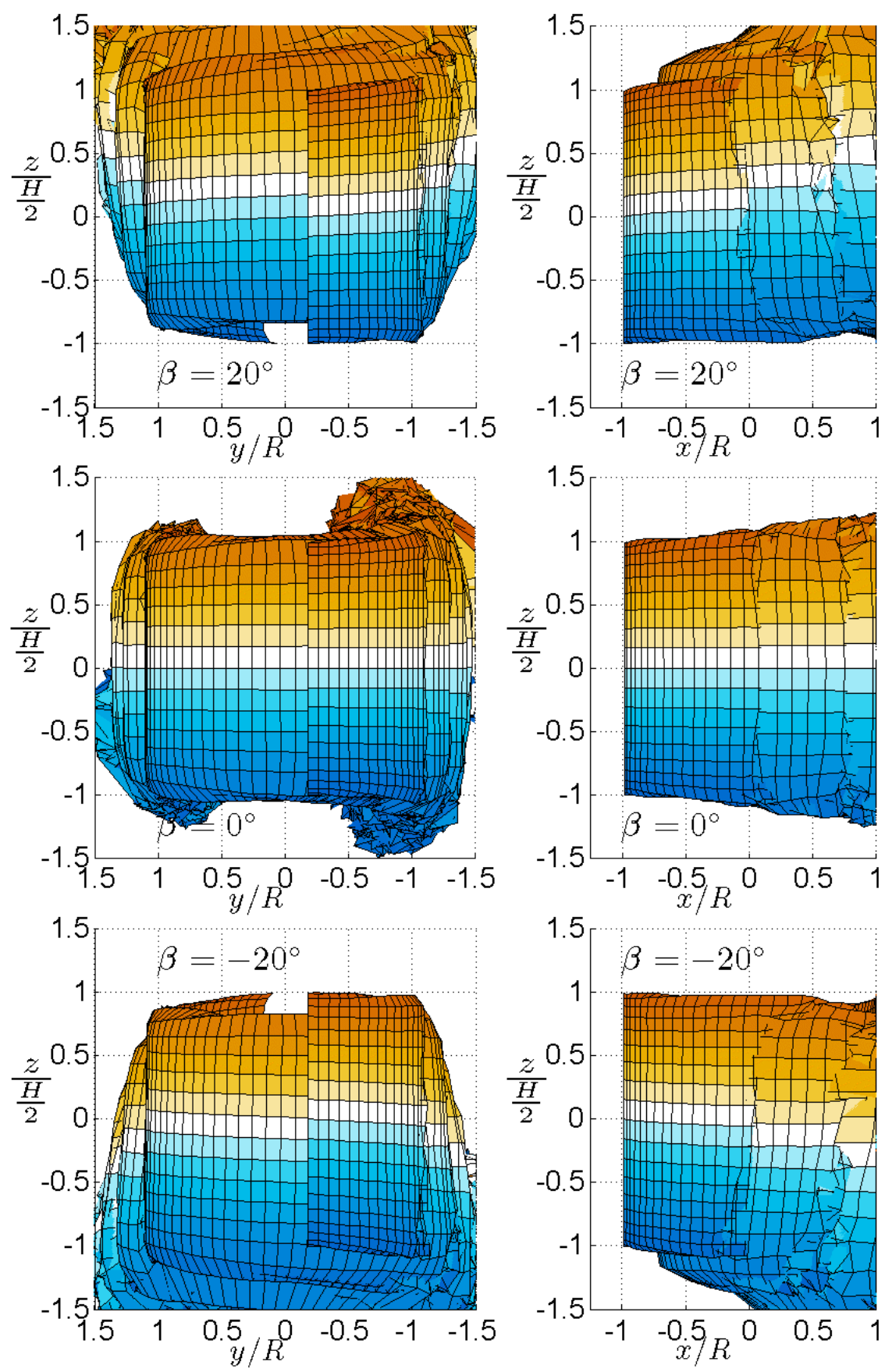

Figure 4: Location of the wake (for $\theta=90^{\circ}$ ) for varying skewed flow angle. 


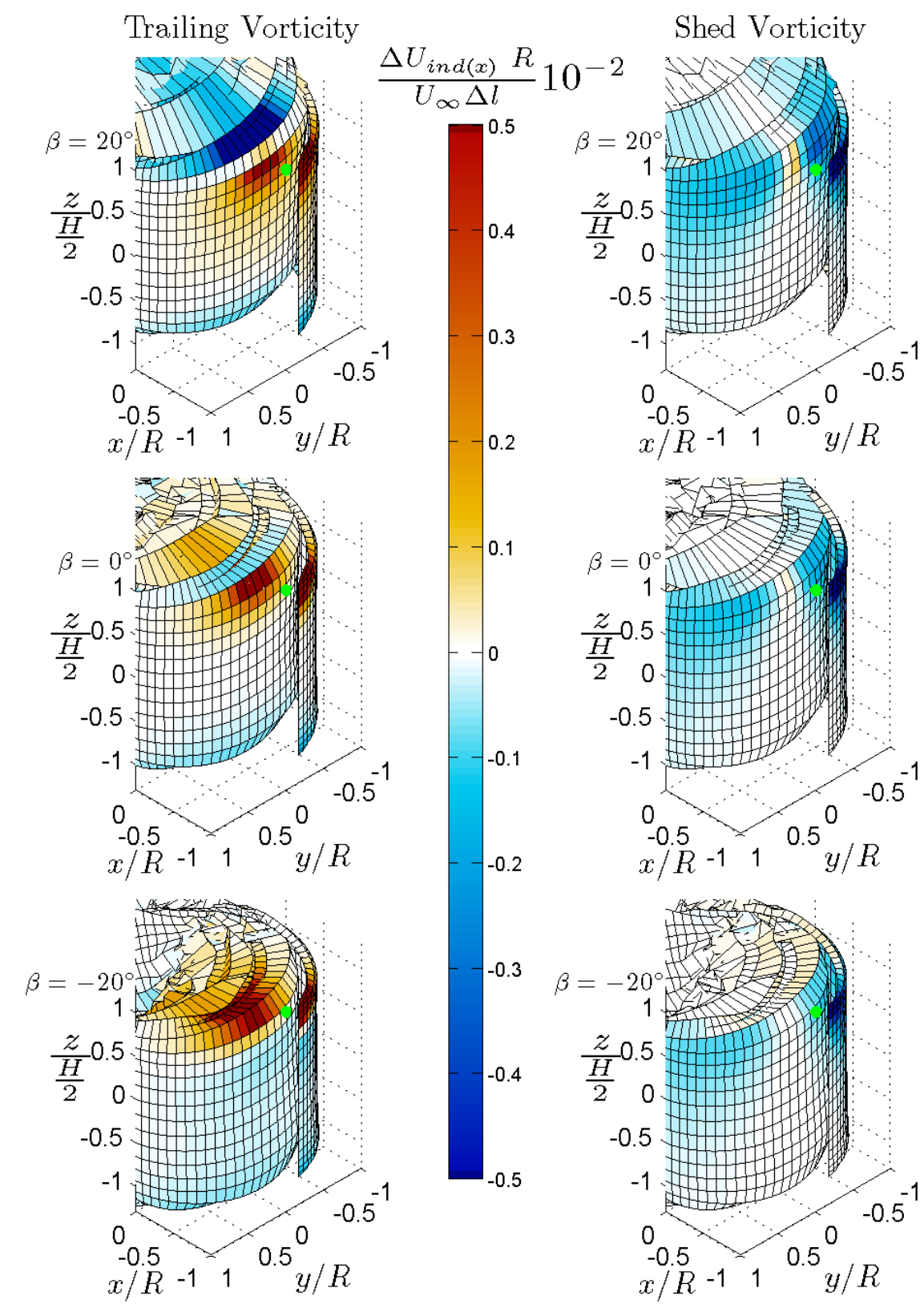

Figure 5: Comparison of induction in $x$ direction at blade tip for varying skewed flow angle. 


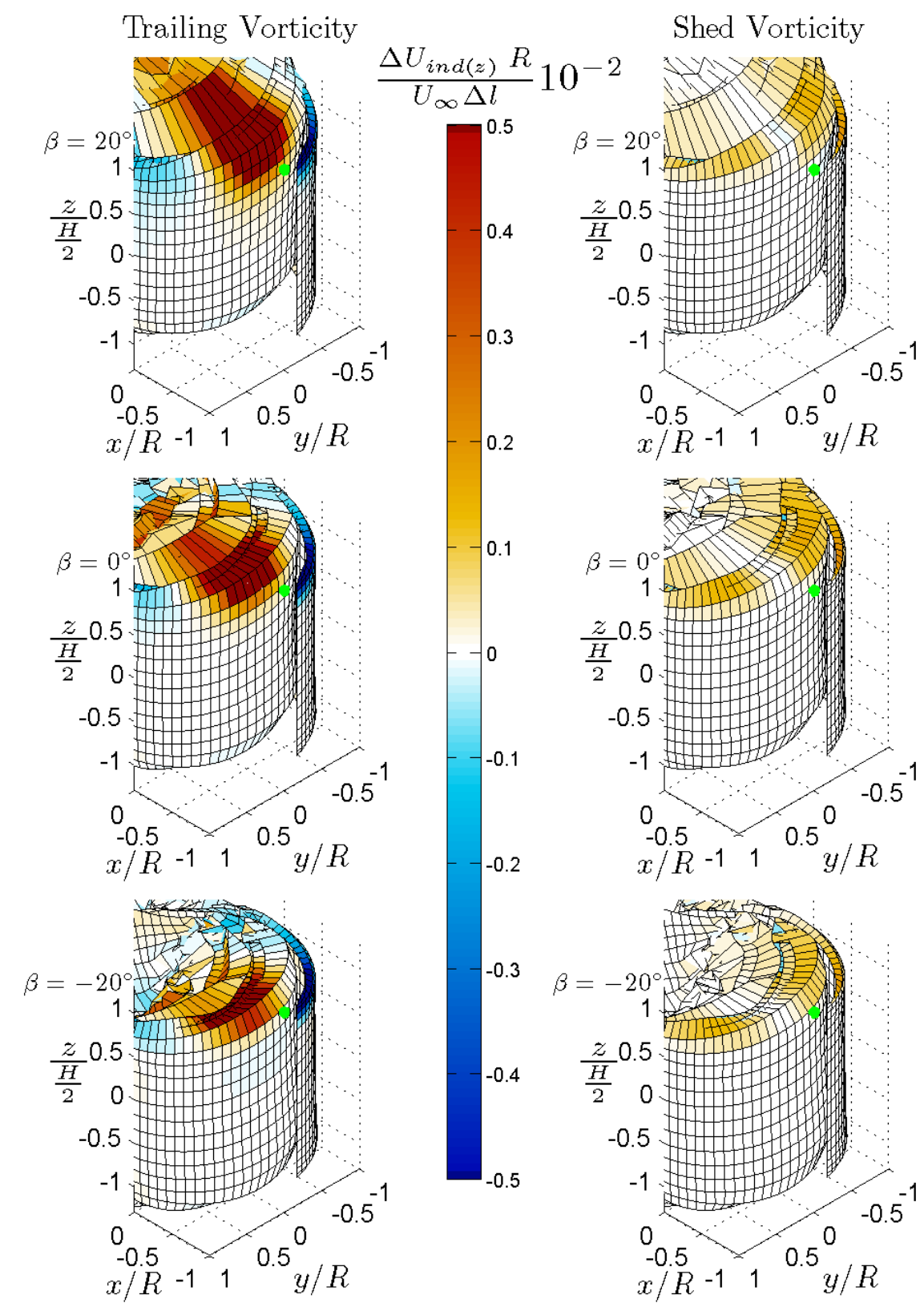

Figure 6: Comparison of induction in $z$ direction at blade tip for varying skewed flow angle. 


\section{B. Induction due to shed vorticity}

The asymmetry of induction caused by the trailing vorticity is reinforced by an asymmetry in induction due to the shed vorticity. The right column of Figure 5 shows the induction due to shed vorticity.

While the trailing vorticity is strongest at tip location, the shed vorticity is strongest at the mid span of the blade (during the upwind passage). The traveling of the wake upward or downward, depending on skew angle, places these strongest shed vortex filaments closer and more aligned with $\frac{2 z}{H}=1, \frac{x}{R}=-1, \frac{y}{R}=0$, thus resulting in a larger or smaller induction.

Due to the roll up of the tip vortex, the shed vortex filaments closest to the tip vortex are rotated and induce an opposite velocity. This interesting process occurs in the numerical simulation, but it is possible to assume that the in real flow, the roll up of the tip vortex would absorb the shed vorticity. It is then important to understand the correct roll up behavior of the tip vortex not only for the induction of trailing vorticity, but also for the induction of shed vorticity.

The effects of shed and trailing vorticity add up, resulting in a larger induction in $z$-direction at the blade tip in the (upward for $\beta=20^{\circ}$ or downward for $\beta=-20^{\circ}$ ).

\section{Influence of the skew angle on the tip vortex movement}

The different induction velocity in $z$ and $x$, combined with a stronger/weaker tip vortex, leads to a different motion of the tip vortex, as seen in the experimental results of Section III.

The locus of the tip vortex, which limits the wake in $z$ direction, has an impact on the region of the downwind passage which operates in the low energy wake flow region and in blade-vortex interaction.

Figure 7a shows the simulation results for the trajectory of tip vortex for the three skew angle cases and different $y$-planes. Following the methodology used for the experimental data of the location of the tip vortices in Section III, Figure $7 \mathrm{~b}$ presents the relative motion of the tip vortices in relation to $U_{\infty}$ for the three skew angles and different $y$-planes.

The results of Figure 7a clearly show the impact of skewed flow in the downwind half of the rotor; due to skew, there is a large region of the downwind blade passage that is operating outside of the wake of the upwind blade passage. Due to the relative motion of the tip vortex, seen in Figure 7b, this region is larger that what is estimated by just taking into account the skew angle of $U_{\infty}$.

The relative motion of the tip vortices derives from two effects;

- The roll of the tip vortex.

- The induction of the wake.

The inability of the panel model to accurately track the complex motion of the roll of the tip vortex explains the difference between the convex and concave trajectories seen in Figures 3b and $7 \mathrm{~b}$.

\section{Validation of effect of skew on tip vortex strength}

Figure 8 presents a comparison between the tip vortex strength simulated with the panel method and the experimental value of circulation obtained by integrating the PIV velocity field. The values of circulation of the tip vortices are non-dimensioned by the circulation of tip vortex element shed at $\theta=90^{\circ}$ for $\beta=0^{\circ}$ (for the simulation and experimental data, respectively).

The experimental values of circulation for planes $y \neq 0$ are corrected taking into account the angle between the direction of the trailing vortex filament and the velocity measurement plane (this corresponds approximately to a relation $\left.\cos ^{\frac{1}{2}}\right)$.

The non-dimensioned experimental and numerical simulation results are in good agreement; the panel model is able to capture the effect of skew on the relative strength of the tip vortex.

The correct relation of tip vortex strength implies that the model is able to capture the effect of skew on the circulation distribution, at least for the upwind region of the rotor.

For the case $\beta=+20^{\circ}$, the agreement between experimental and numerical results is lower than for the other two skew angles. This can have three sources:

- Lower quality of the experimental data, with an undetected bias.

- The roll up process of the trailing vorticity, at the blade tip region, for the $\beta=+20^{\circ}$ case, entails a larger amount of inboard vorticity than in the two other skew cases. 


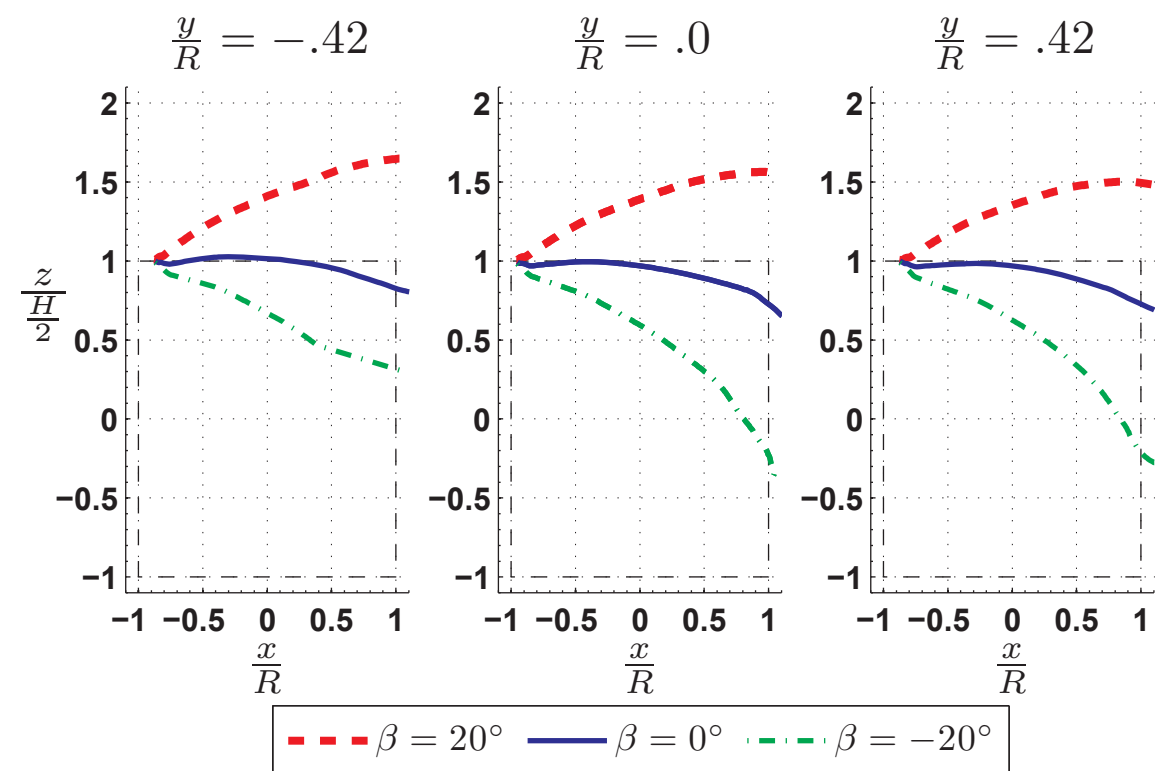

(a) Tip vortex locus (absolute).

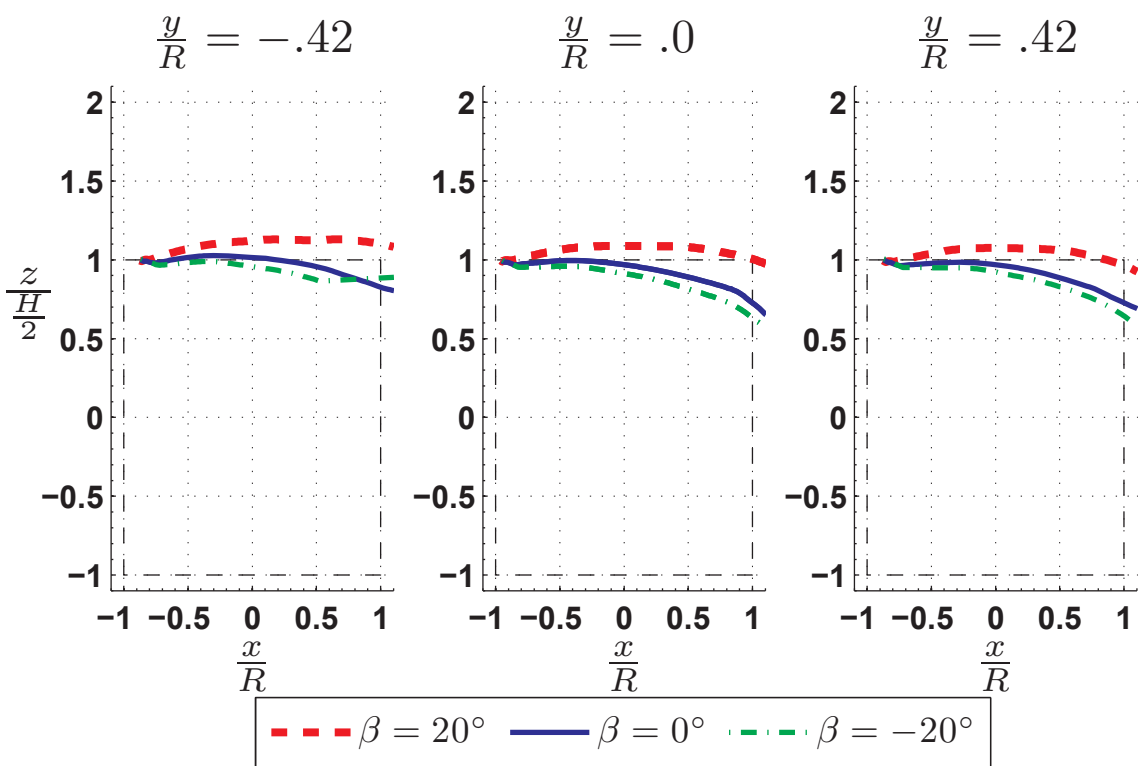

(b) Tip vortex locus relative to $U_{\infty}$ direction.

Figure 7: Tip vortex locus (numerical simulation data) for $\beta=+20^{\circ}, 0^{\circ}$ and $-20^{\circ}$, at the planes $\frac{y}{R}=-.42$, 0 and .42 . 
- There is an important $3 D$ viscous effect that causes an extra relative generation of lift at the tip for $\beta=+20^{\circ}$.

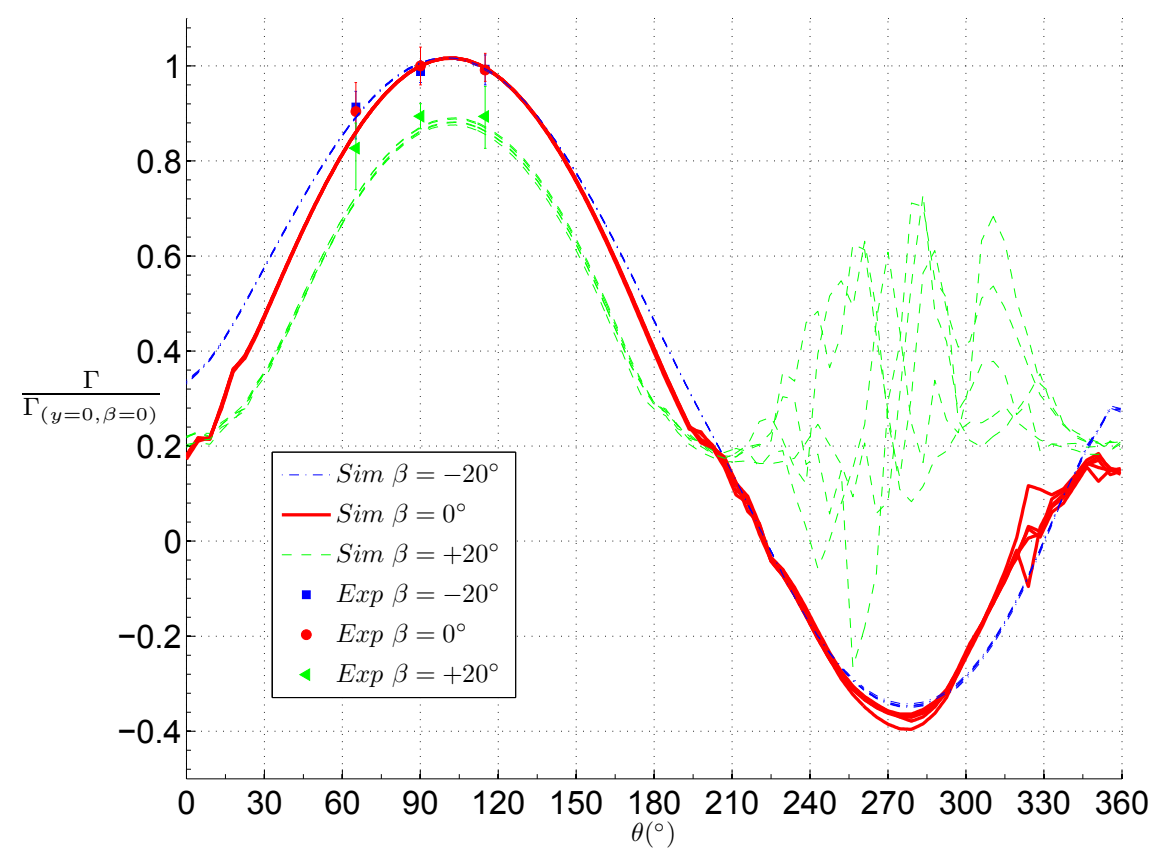

Figure 8: Comparison between simulation and experiment of strength of tip vortex over azimuthal angle.

The numerical results for $\beta=+20^{\circ}$ show a high variability of the strength of the tip vortex for $210^{\circ}<$ $\theta<360^{\circ}$, which is a result of the blade vortex interaction between the blade tip and the wake shed during the upwind passage of the blade. This effect will be further discussed in Section VI.

\section{Effect of skew in bound, trailing and shed vorticity}

In Section $\mathrm{V}$ we discussed how the variation, with skew angle, of the position of the wake in $z$, causes an asymmetric induction field over the blade, where we took the blade tip region as an example.

The effect of skew angle on the induction at the blade tip region implies also an effect on the strength of the tip vortex. In Section D, the results of the numerical simulation, regarding the effect of skew angle on the strength of the tip vortex, were validated by the wind tunnel experimental results with PIV.

The observed effect on the strength of the tip vortex implies an obvious effect of skew angle on the trailing vorticity distribution, and, by inference, on the shed vorticity. In this section, we will use the results from the numerical simulation to observe the effect of skew on trailing and shed vorticity distribution of the wake.

\section{A. Trailing vorticity}

Figure 9 presents three plots, for three skew angles, of the strength of the trailing vorticity shed at each azimuthal position over the span of the blade. Figure $9 \mathrm{~b}$ shows the trailing vorticity distribution for $\beta=0^{\circ}$; the distribution of vorticity for skew angles $\beta=20^{\circ}$ and $-20^{\circ}$ are shown respectively in Figures 9a and 9c.

The distribution for skew angles $\beta=20^{\circ}$ and $-20^{\circ}$ are naturally (almost) symmetric in span direction and magnitude. The spanwise asymmetry of the strength of the trailing vorticity during the upwind passage of the blade was already interpreted as an effect of the asymmetry of the induction from the wake due to the asymetry of the location and strength of the wake.

The wake generated in the downwind passage of the wake $\left(180^{\circ}<\theta<360^{\circ}\right)$ is more complex and it is the source of the positive effect of skew in power conversion observed in, ${ }^{12}$ and discussed in. ${ }^{3}$ Because the $\beta=20^{\circ}$ and $-20^{\circ}$ cases are symmetric, we will discuss only the downwind results for $\beta=0^{\circ}$ and $\beta=20^{\circ}$. 
For $\beta=0^{\circ}$, the downwind passage trailing vorticity distribution can be divided in three regions, resulting from the location of the intersection between the downwind blade passage and the wake generated during the upwind passage. Figure 9b presents four areas of strong release of trailing vorticty for the azimuth $180^{\circ}<$ thet $a<360^{\circ}$. These regions are located at the spanwise positions of the blade tips $\left(\frac{z}{H / 2}=1\right.$ and $\left.\frac{z}{H / 2}=-1\right)$ and at the spanwise location near the interaction of the blade with the tip vortices generated during the upwind rotation; this is results in Figure $9 \mathrm{~b}$ as a triangular distribution of released trailing vorticity with a maximum at $\theta \approx 270^{\circ}$ and $\frac{z}{H / 2} \approx \pm 0.7$.

\section{B. Bound vorticity}

Figure 11 shows the distribution of the bound vorticity along span, over the rotation of the blade (similar to Figure 9 for the trailing vorticity).

Comparing the upwind half $\left(0^{\circ}<\theta<180^{\circ}\right)$ of the three subplots of Figure 11, the effect of skew is visible in the spanwise asymmetry of the bound vorticity. In Section V, we discussed the effect of skew angle as generating an asymmetry of the induction at the tip location. Extending this argument to the entire span, the asymmetry (with skew angle) of the bound vorticty in the upwind half of the rotation is a consequence of the asymmetry of the induction by the wake.

The more windward half of the span experiences a lower induction and thus a higher bound vorticty. If the asymmetry in the upwind half of the rotation is easily explained by the asymmetry in induction, the downwind half of the rotation presents assymetry not only in terms of spanwise distribution, but also in the sign of the bound circulation.

The bound circulation is composed of two components, one the result of the translational and pitching motion of the blade, and the other due to the local wind field. During most of the upwind half of the rotation, the two components of circulation have the same sign; however, during the downwind half, the two components of circulation have usually opposite sign. The regions in the downwind half where the value of bound circulation has the same sign as in the upwind half (in the case of Figure 11, positive), are regions where the local induction is very high and thus, the circulation due to the local wind velocity is small or even, if the local induction is larger than one, of the same sign as the circulation due to the uniform motion of the blade.

To understand the distribution of the bound vorticity during the downwind passage, and the effect of skew in that distribution, we must first understand the distribution of induction during the downwind passage, and the effect of skew on that induction distribution.

Figure 10 plots the spanwise induction distribution at the $1 / 4 c$ position of the blade at three azimuthal positions $\theta=225^{\circ}, 270^{\circ}$ and $315^{\circ}$, for two skew angles $\beta=0^{\circ}$ and $+20^{\circ}$. The plots are divided in induction in $x$ direction due to shed vorticity in the wake $\left(U_{i n d(x)}\right)$, induction in $x$ direction due to trailing vorticity in the wake $\left(U_{i n d(x)}\right)$, and total flow velocity in $x$ direction $U_{x}$ (wake induction and $U_{\infty}$ ).

The skewed flow results in a spanwise asymmetry of the induction due to shed vorticity. Figure 10 shows that the induction due to shed vorticity for $\beta=20^{\circ}$ is small for the region not operating in the wake generated during the upwind passage, due to the location of those wake segments. The induction, due to shed vorticity, in this region, is the result of the near wake generated during the downwind passage, both the current new near wake, and the wake portions that were previously generated during the downwind passage.

This near wake shed vorticity is stronger in the region of the downwind passage that is cleared from the upwind wake, in comparison with the spanwise region in blade wake interaction. This effect is visible in Figure 13, which we will discuss further ahead.

The induction due to the trailing vorticity is also affected by the skew angle. Due to skew, the lower trailing vortex, generated during the upwind blade passage, interacts with the blade close to mid span. As in the $\beta=0^{\circ}$ case, the region, defined between the tip vortices generated upwind, experiences an added induction (deceleration); however, in the region exterior to the location of the tip vortices, the flow is accelerated.

The combined results of the induction by shed and trailing vorticity is seen in the further right side column of Figure $10\left(U_{x}\right)$. The skewed flow results in a lower half span operating in a very low induction factor, and an upper half operating in a large induction factor flow.

This asymmetry of induction, when compared to the $\beta=0^{\circ}$ case, explains the added power conversion during the downwind blade passage, which contributes to the positive effect on power conversion detected on VAWTs in skew. 


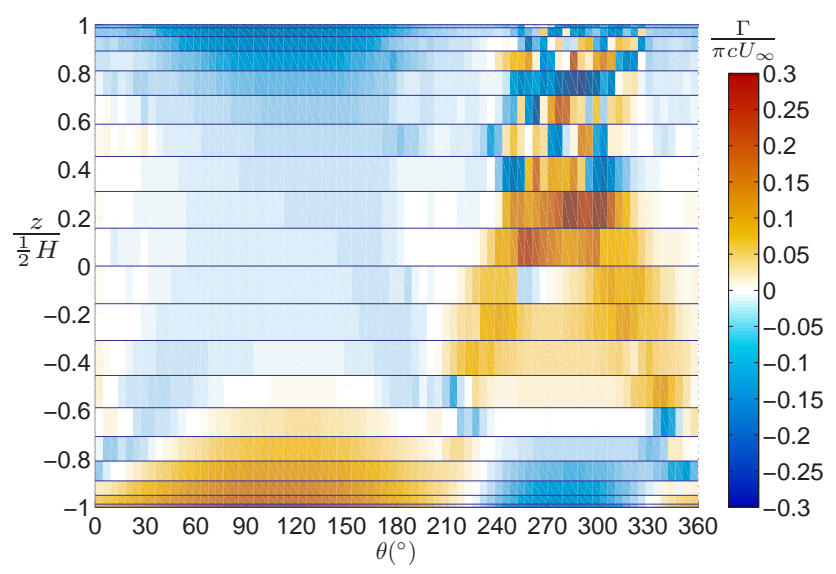

(a) $\beta=+20^{\circ}$.

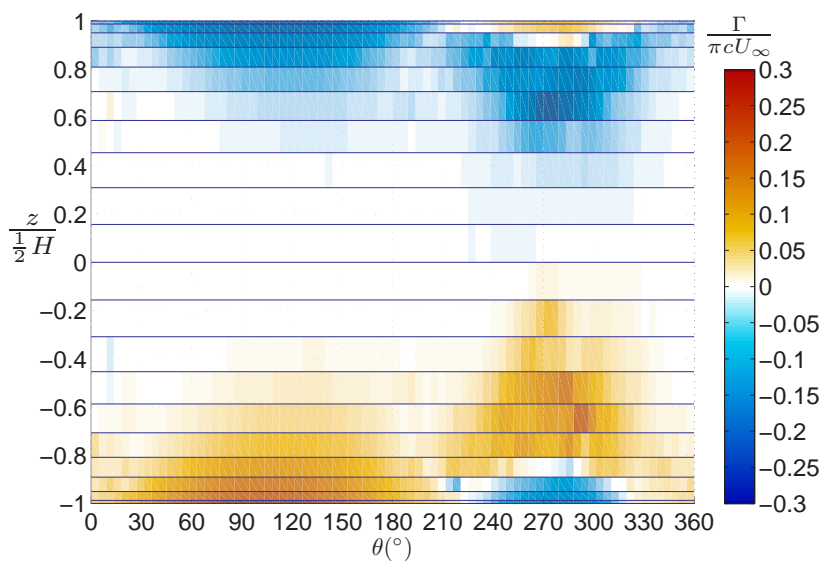

(b) $\beta=0^{\circ}$.

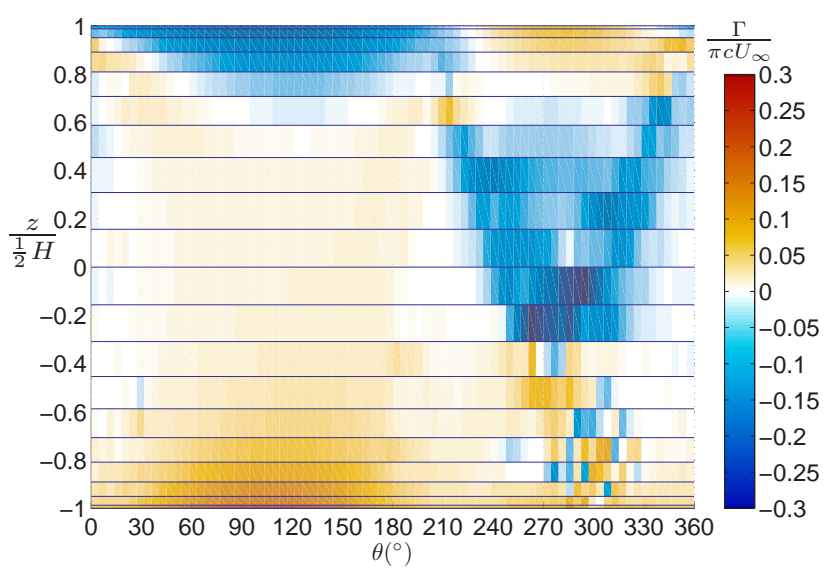

(c) $\beta=-20^{\circ}$.

Figure 9: Trailing vorticity shed at each azimuthal position, over span, for $\beta=+20^{\circ}, 0^{\circ}$ and $-20^{\circ}$. 


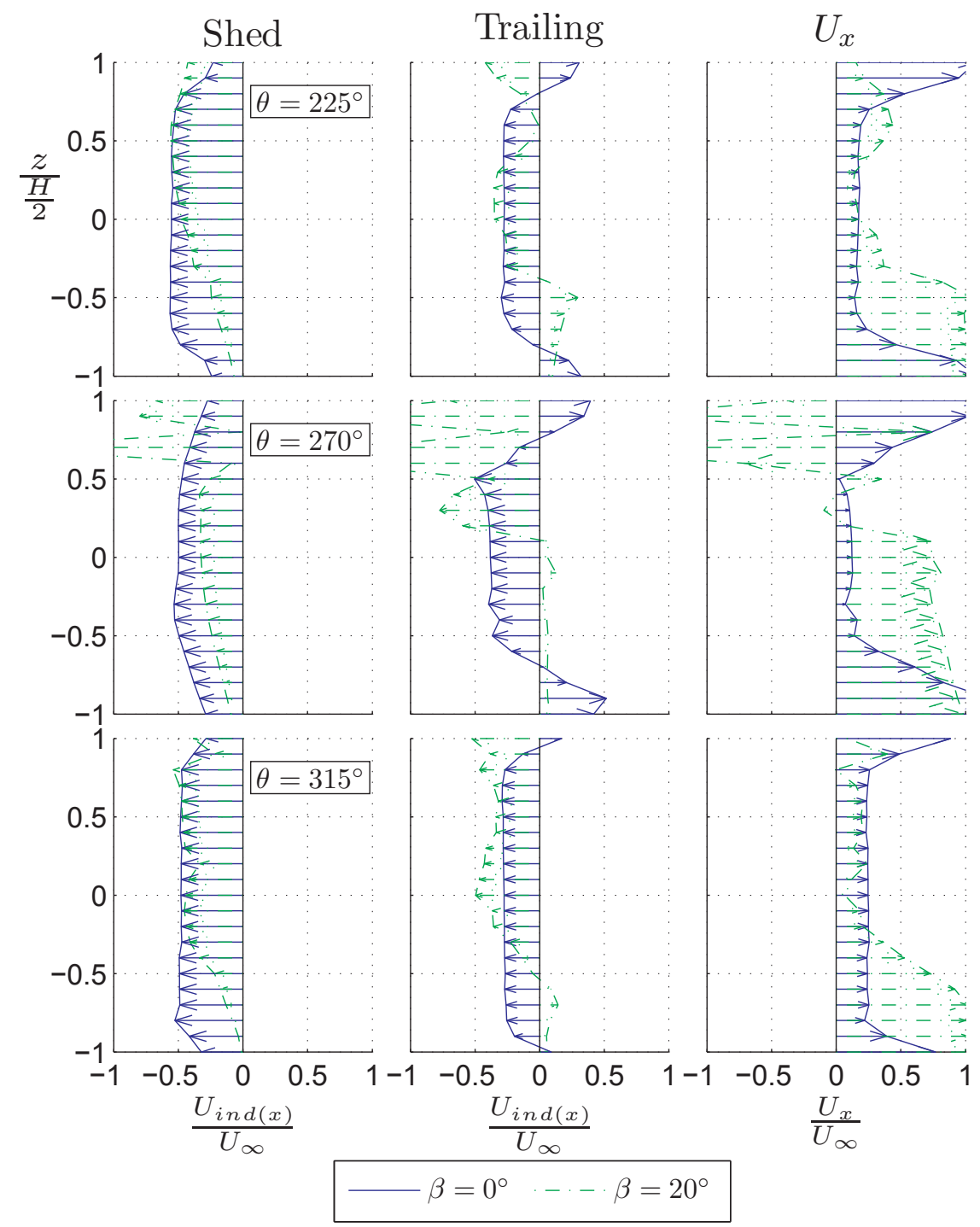

Figure 10: Wake induction over span at three downwind azimuthal positions $\theta=225^{\circ}, 270^{\circ}$ and $315^{\circ}$, for two skew angles $\beta=0^{\circ}$ and $+20^{\circ}$. 
The total induction in the upper half of the blade for $\theta=270^{\circ}$ shows, in the simulation, an unsteady induction above 1 . Although some of this result is clearly a consequence of the numerical implementation, it is important to understand the implication of the spanwise varying bound vorticity.

During the downwind passage, the circulation due to the pitching motion of the airfoil, and the circulation due to the local wind speed, are in opposition of sign. The sign/ direction of the total bound circulation is then a result of the magnitude and direction of the local wind speed.

Figure 11, taking into account the discussion of the local induction (Figure 10), clearly shows the impact of the magnitude of the downwind passage induction on the direction/magnitude of the bound vortex.

In parallel to the effect on the generation of torque, the spanwise variation of the direction of the bound vortex implies a spanwise variation on the direction of the normal force; the normal force is, for a bound vortex in potential flow (neglecting added mass effects), proportional to the product of the bound circulation with the tangential component of the perceived velocity. Figure 12 shows the normal force distribution over span at each azimuthal position, for $\theta=20^{\circ}$ and $0^{\circ}$. The normal force distribution of Figure 12 follows in sign, as stated previously, the bound vorticity strength distribution of Figure 11.

\section{Shed vorticity}

The shed vorticity release at each time step follows the time/azimuthal variation of the bound vorticity. From the previous analysis of Figure 11, it is possible to understand the results for the shed vorticity distribution in Figure 13.

The shed vorticity is of extreme importance on the VAWT, since it is the result of the energy exchange process, contrary to the trailing vorticity, which is an inefficiency of the system. Because the system is periodic, the total shed vorticity during one period must be zero. The exchange of energy is not determined by the the amount of shed vorticity, but by the amplitude of the magnitude of the shed vorticity.

The larger amplitude of bound vorticity in some of the regions of the VAWT leads to the larger amplitude of the shed vorticity at each azimuthal position seen in Figure 13, and to the larger efficiency of the VAWT in skew. However, this amplitude decreases once again for higher skew angles.

\section{Validation with experimental torque wind tunnel measurements}

In the previous sections we validated the panel model results for the effect of skew on the strength and location of the tip vortices in the near wake, comparing the simulation values with PIV measurements.

In this section, we compare the effect of skew angle on the total torque of the rotor, comparing the results from the vortex model with the experimental wind tunnel measurements in ${ }^{1}$ and. ${ }^{2}$

In the results presented in ${ }^{1}$ and,$^{2}$ a small H-Darrieus rotor is placed at different skew angles in an open-jet wind tunnel. By varying torque and tip-speed ratio, a curve of the maximum power coefficient measured at each skew angle was found.

The model is a two straight bladed NACA $0018 \mathrm{H}$-Darrieus, with blade chord $0.08 \mathrm{~m}$. The model has a height of $0.5 \mathrm{~m}$ and $0.375 \mathrm{~m}$ radius.

In the current comparison, the simulations of the model at different skew angles is performed at a constant tip speed ratio of 3.1 , for $\theta=0^{\circ}, 10^{\circ}, 20^{\circ}$ and $30^{\circ}$. In the experimental work $\left({ }^{1}\right.$ and $\left.^{2}\right)$, the tip speed ratio, for the range of skew angles, varies from 2.9 to 3.2 ; the experimental results in ${ }^{2}$ also show that power coefficient is fairly constant between tip speed ratios 2.9 until 3.2. Thus, the option in the simulations of not varying the speed ratio according to the experiments should have a small impact on the analysis of the effect of skew angle.

An additional reason for not varying the tip speed ratio with the potential flow panel model simulations, is the inability of the model to account for the increasing power loss with increasing tip speed ratio due to drag, which occurs in a real VAWT. In a panel model simulation of the VAWT, the increasing tip seed ratio will result in an increasing power coefficient, converging to the theoretical maximum.

Following the methodology in the previous sections, Figures 14, 15 and 16 show, respectively, the bound, shed and trailing vorticity over the span at each azimuthal position.

The model in this simulation, used in the work of, ${ }^{3}{ }^{1}$ and, ${ }^{2}$ has a larger $R / H$ ratio than the model described in the previous section. This larger $R / H$ ratio implies a differen location of the interaction during the downwind passage of the blade and the wake generated during the upwind passage. 


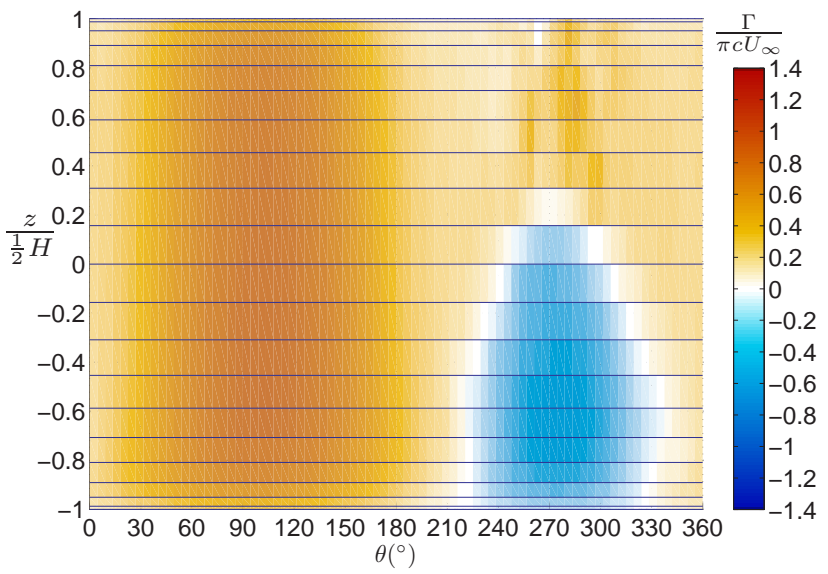

(a) $\beta=+20^{\circ}$.

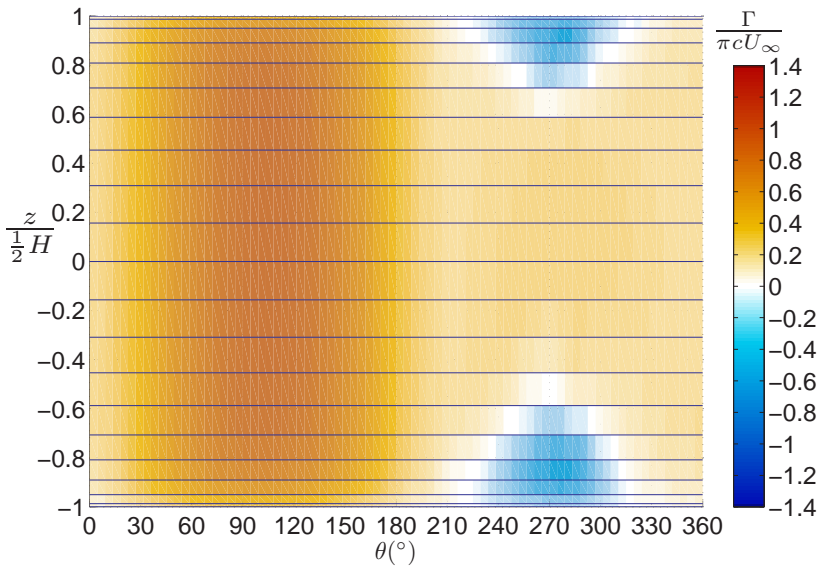

(b) $\beta=0^{\circ}$.

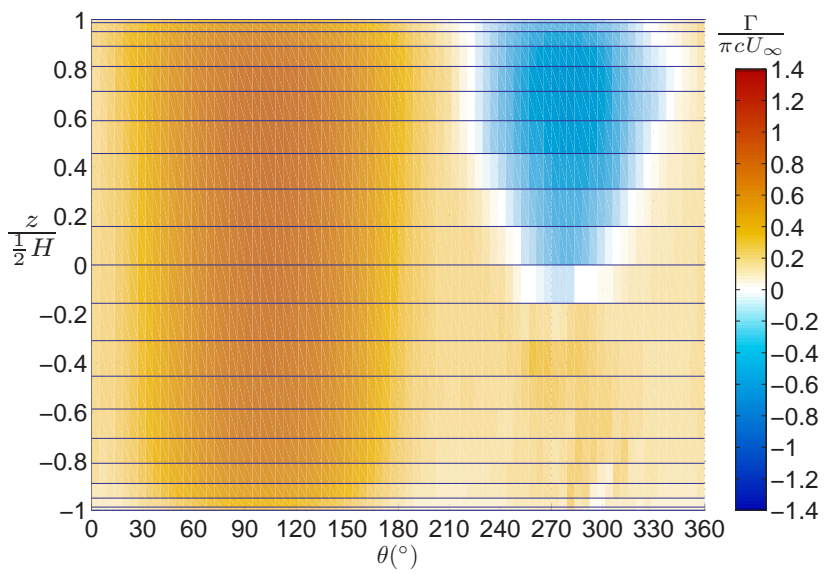

(c) $\beta=-20^{\circ}$.

Figure 11: Bound vorticity at each azimuthal position, over span, for $\beta=+20^{\circ}, 0^{\circ}$ and $-20^{\circ}$. 


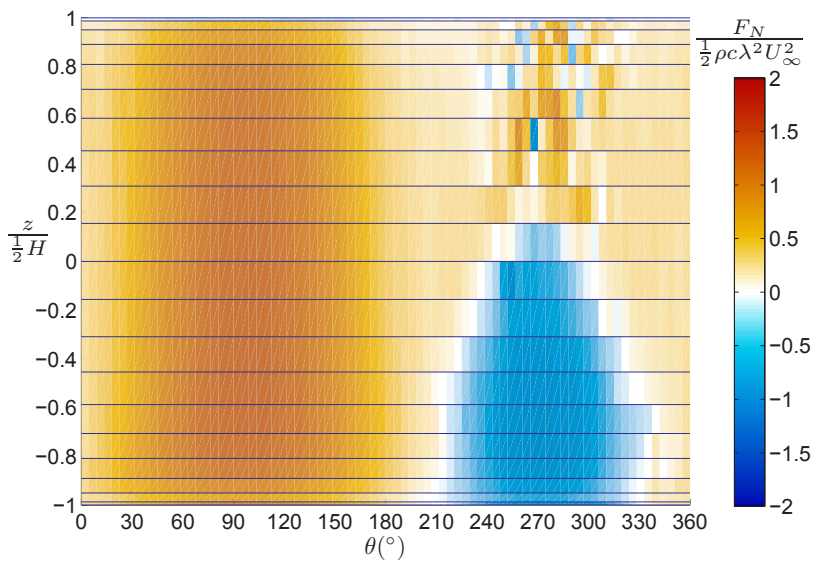

(a) $\beta=+20^{\circ}$.

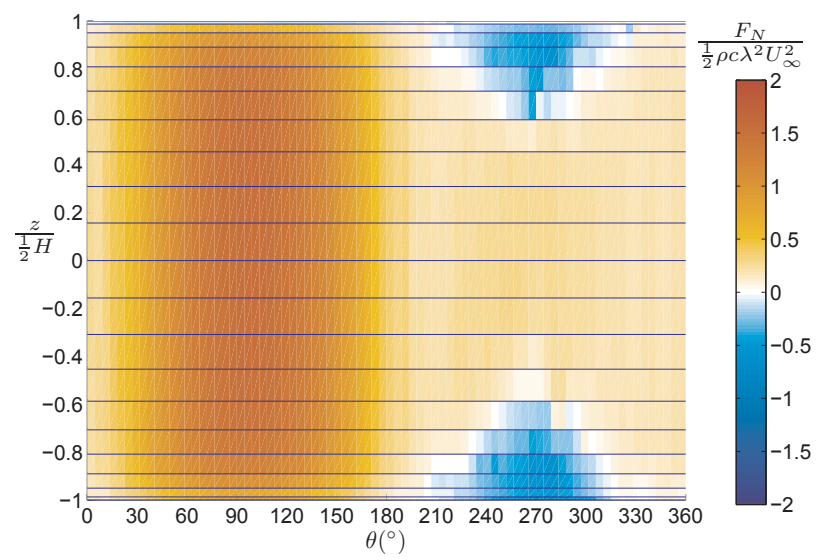

(b) $\beta=0^{\circ}$.

Figure 12: Normal force at each azimuthal position, over span, for $\beta=+20^{\circ}$ and $0^{\circ}$. 


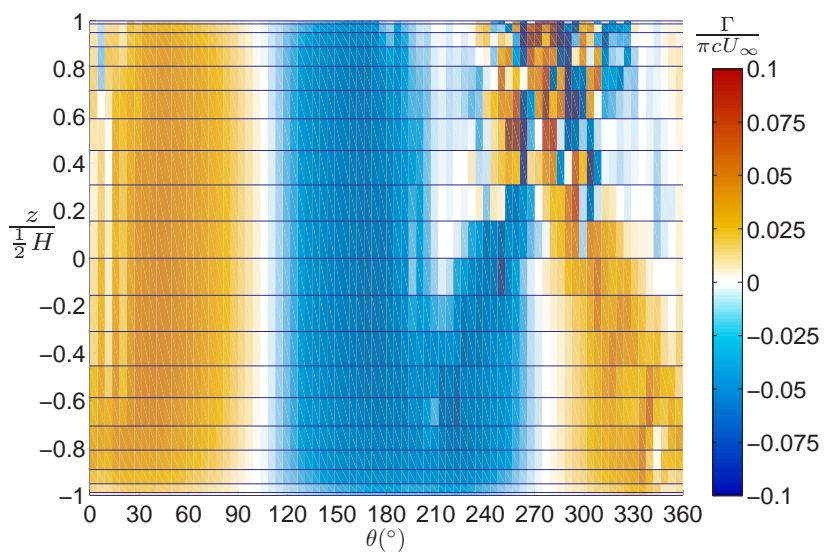

(a) $\beta=+20^{\circ}$.

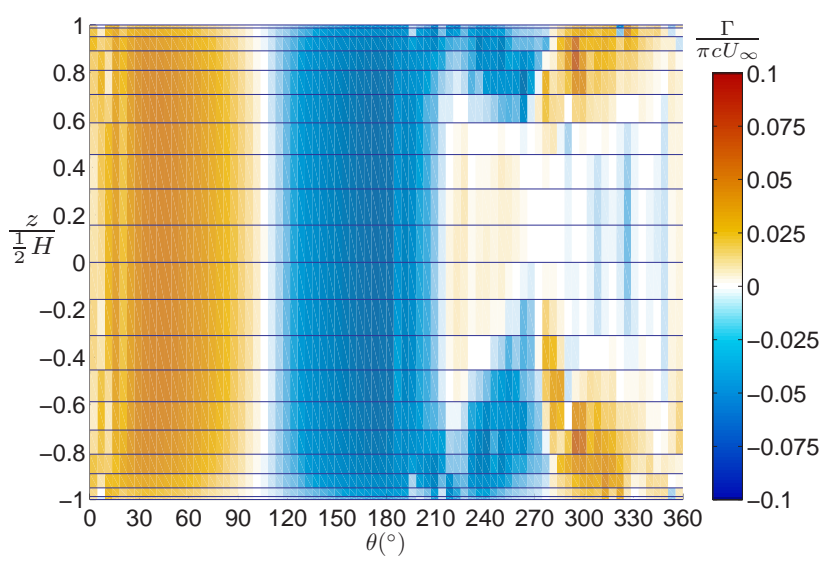

(b) $\beta=0^{\circ}$.

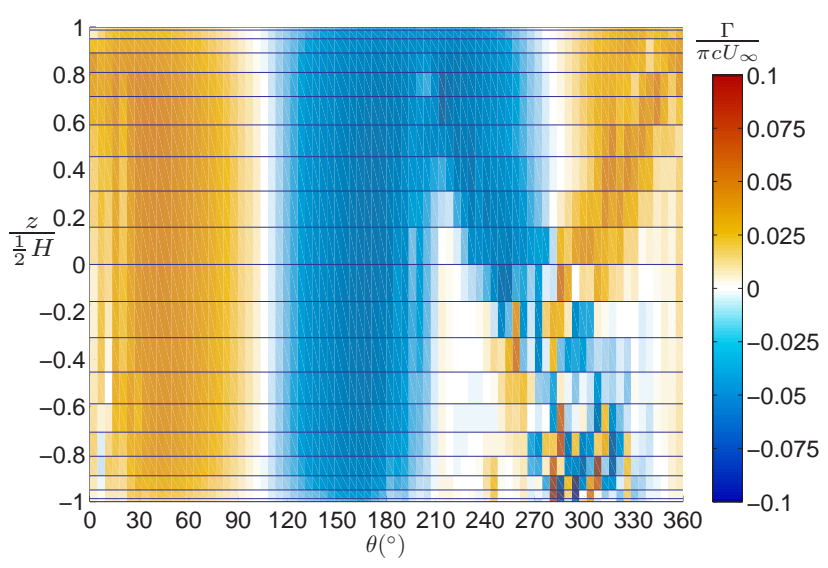

(c) $\beta=-20^{\circ}$.

Figure 13: Shed vorticity shed at each azimuthal position, over span, for $\beta=+20^{\circ}, 0^{\circ}$ and $-20^{\circ}$.

19 of 25 
The maximum added power due to skew angle increases with increasing $R / H$ ratio, due to the relatively larger area of downwind passage sweep that operates outside the wake generated during the upwind passage.

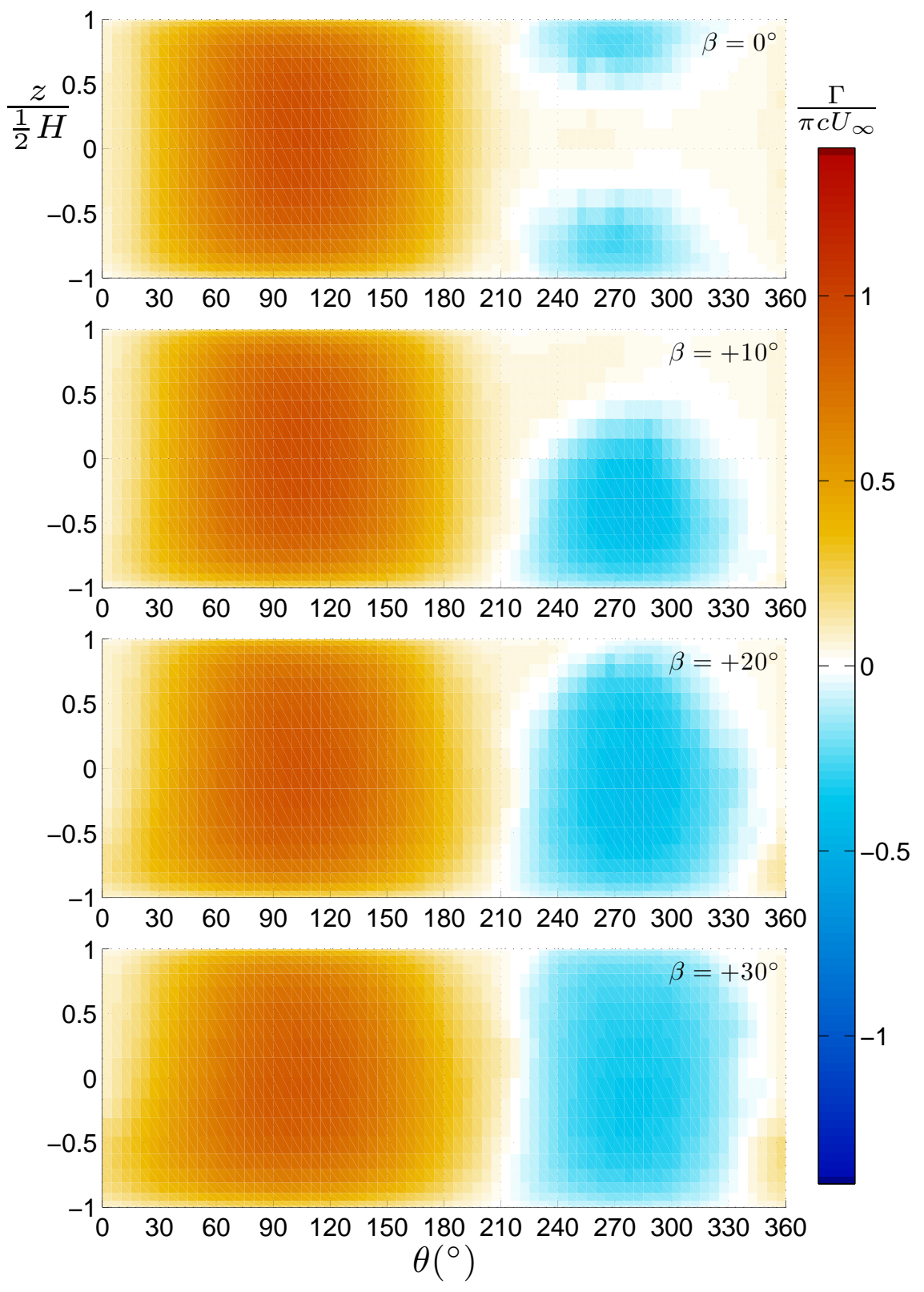

Figure 14: Bound vortex over blade span at each azimuthal position, for $\beta=0^{\circ}, 10^{\circ}, 20^{\circ}$ and $30^{\circ}$.

Figure 17 shows the effect of skewed angle on torque distribution.

Figure 18 shows a comparison of relative power coefficient as a function of skew angle, comparing the results of the simulation with the panel model and the experimental results from ${ }^{1}$ and. $^{2}$

The results of the simulation capture the trend observed in the experiment. This result raises a question regarding what is the effect of skew on the drag force on the blade. Since the model can only capture the increase with skew of inviscid torque, the fact that the model still captures reasonably the experimentally observed effects, implies that: either the viscous drag is small compared with the tangential component of lift (and thus fall in the range of uncertainty of the experiment and gap between simulation and experiment, including tip speed ratio difference); or the increase of the tangential component of lift is met with a linear increase of drag. Further experiments and simulations will be necessary to evaluate these two hypothesis.

20 of 25 


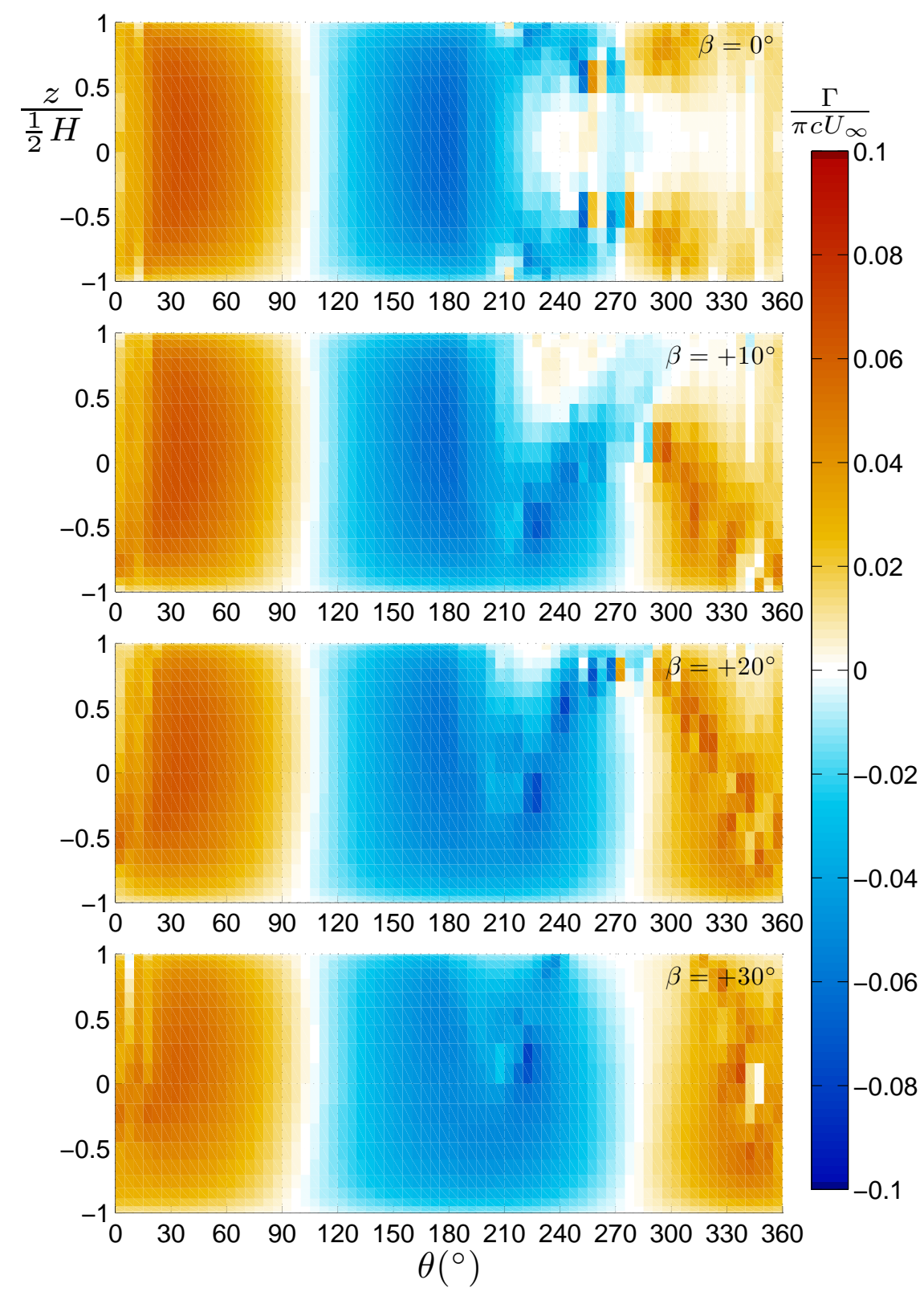

Figure 15: Shed vorticity at each azimuthal position, for $\beta=0^{\circ}, 10^{\circ}, 20^{\circ}$ and $30^{\circ}$. 


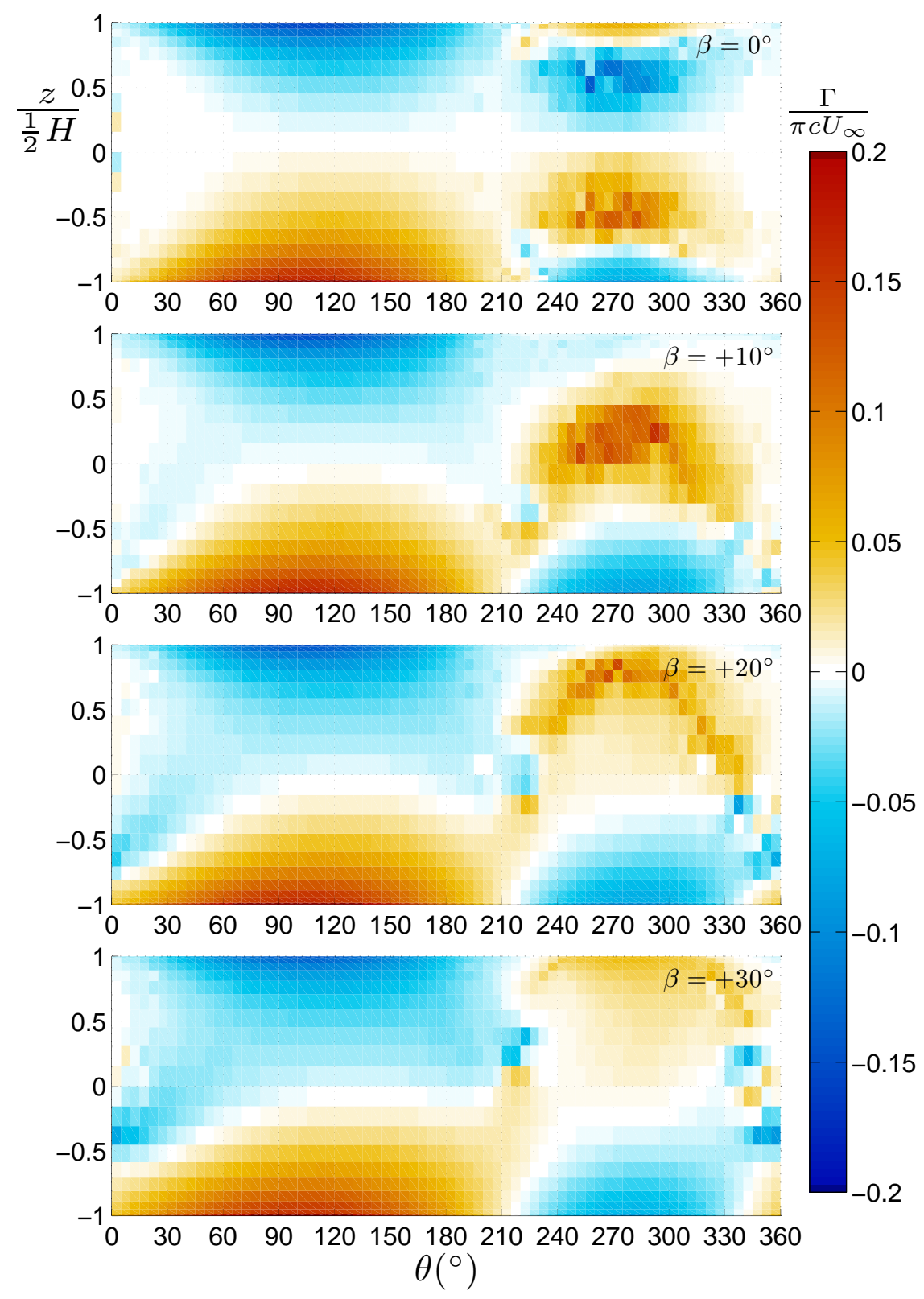

Figure 16: Trailing vorticity at each azimuthal position, for $\beta=0^{\circ}, 10^{\circ}, 20^{\circ}$ and $30^{\circ}$. 


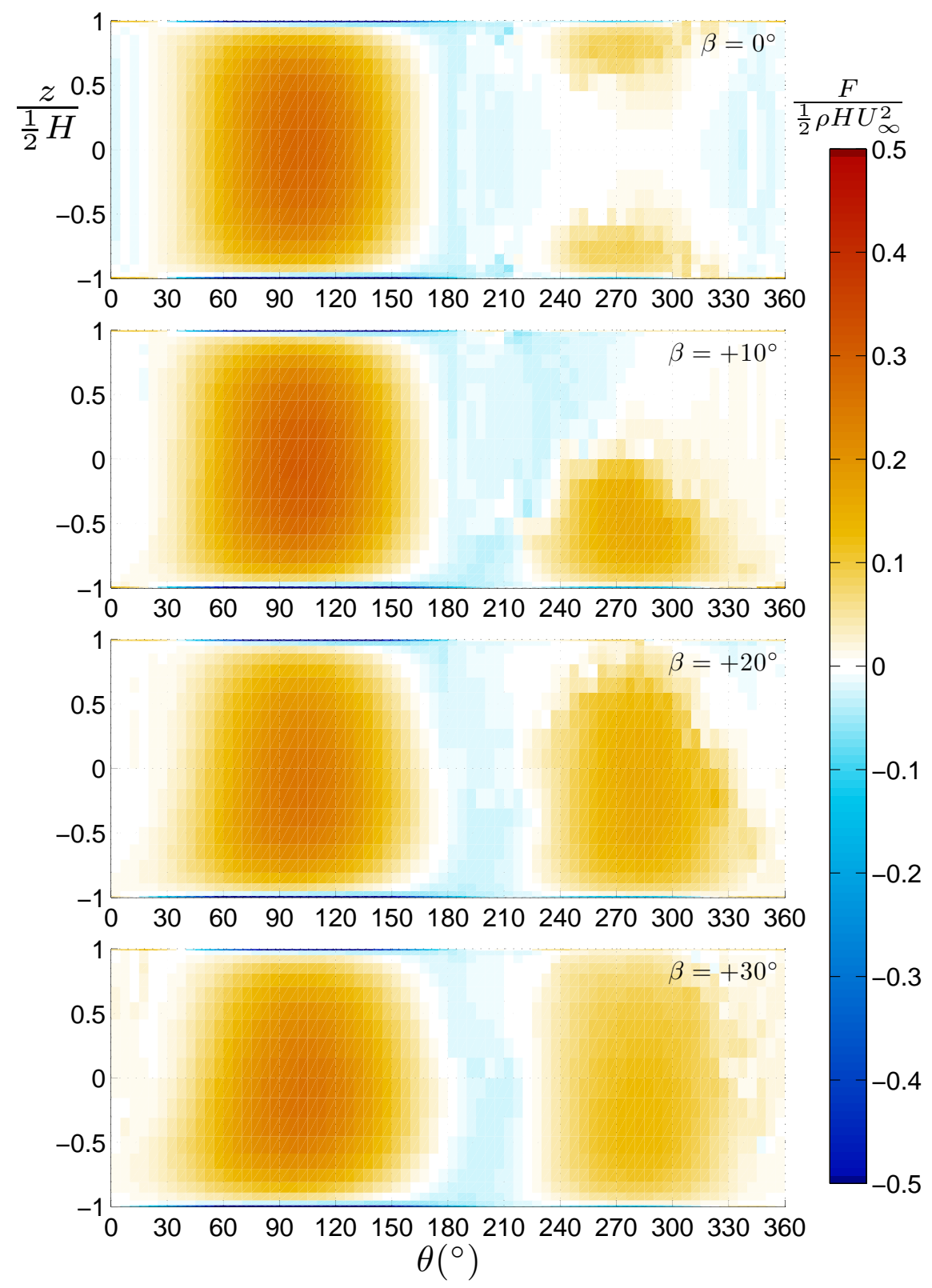

Figure 17: Tangential force distribution over span at each azimuthal position, for $\beta=0^{\circ}, 10^{\circ}, 20^{\circ}$ and $30^{\circ}$. 


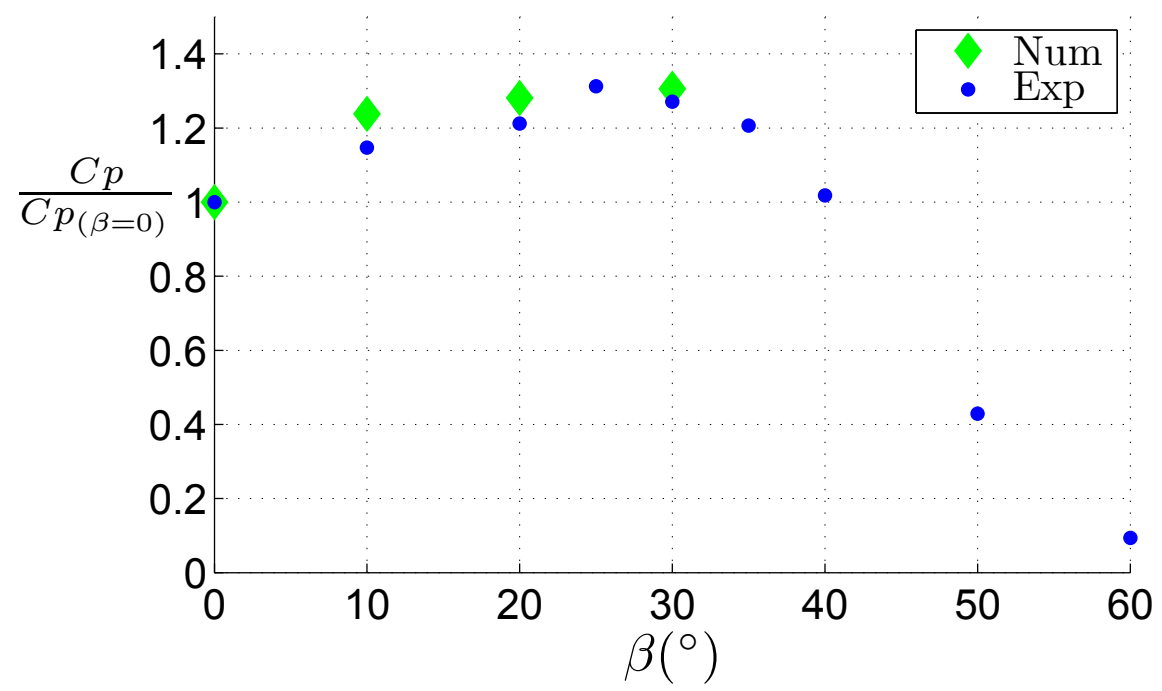

Figure 18: Comparison of variation of power with skew angle, experiments $\left({ }^{1} \mathrm{and}^{2}\right)$ vs. simulation.

\section{Conclusions}

Skewed flow increases the performance of an H-VAWT by modifying the region of interaction of the downwind blade passage with the upwind generated wake.

The skew angle causes an asymmetry of the wake in $z$-direction, both in terms of position, strength of the shed and trailing vorticity, and expansion of the wake in $y$-direction.

The asymmetry with skew angle was verified with wind tunnel experiments using Particle Image Velocimetry; the PIV results for the tip vortex in the near wake allow to quantify the effect of skew angle in the strength of the tip vortex, and its convection.

The 3D unsteady free wake panel code is able to replicate the effect of skew in the relative strength of the tip vortex, as validated with the experimental results for circulation of the tip vortices. The panel model was not able to capture the roll-up motion of the tip vortex, which can be attributed to the model's first order Newton solution of the displacement of the vortices

Using the panel code, the simulation results showed the impact of skew angle in the strength of the wake, both trailing and shed vorticity. Also, the effect of skew in both bound vorticity, normal and tangential force was shown.

The extra power obtained in skewed flow comes from that, with increasing skew angle, an increasing area of the downwind blade passage is operating outside the upwind generated wake, thus experiencing an incoming flow with larger energy content.

However, this is traded off by a decreasing component of the flow which is normal to the blade's lifting line. This decreasing effect is not uniform in spanwise direction, due to the fact that the induction of the wake is asymmetric in spanwise.

\section{Bibliography}

\section{References}

${ }^{1}$ S. Mertens, G. van Kuik, and G. van Bussel. Performance of a H-Darrieus in the skewed flow on a roof. Journal of Solar Energy Engineering, 125:433-440, 2003.

${ }^{2} \mathrm{~S}$. Mertens. Wind energy in the built environment: concentrator effects of buildings. PhD thesis, Delft University of Technology, 2006. 
${ }^{3}$ C.J. Simão Ferreira, G. van Bussel, and G. van Kuik. Wind tunnel hotwire measurements, flow visualization and thrust measurement of a VAWT in skew. Journal of Solar Energy Engineering, 128(4):487-497, November 2006.

${ }^{4} \mathrm{C}$. Hofemann. Investigation on tip vortex evolution of a vertical axis wind turbine with stereo piv. Master's thesis, Faculty of Aerospace Engineering, Delft University of Technology, Mai 2008.

${ }^{5}$ C. Hofemann, C.J. Simão Ferreira, K. Dixon, G. van Bussel, G. van Kuik, and F. Scarano. 3d stereo piv study of tip vortex evolution on a VAWT. In EWEC 2008 - European Wind Energy Conference - Brussels, 2008.

${ }^{6} \mathrm{~K}$. Dixon. The near wake structure of a vertical axis wind turbine -including the development of a 3D unsteady free-wake panel method for VAWTs. Master's thesis, Faculty of Aerospace Engineering, Delft University of Technology, April 2008.

${ }^{7}$ K. Dixon, C.J. Simão Ferreira, C. Hofemann, G.J.W. van Bussel, and G.A.M. van Kuik. A 3D unsteady panel method for vertical axis wind turbines. In T Chaviaropoulos, editor, The proceedings of the European Wind Energy Conference and exhibition EWEC 2008 Brussels, 2008. 